\title{
Numerical Solutions of Elliptic Partial Differential Equations by Using Finite Volume Method
}

\author{
Eyaya Fekadie Anley
}

Department of Mathematics, College of Natural and Computational Science, School of Graduate Studies, Haramaya University, Haramaya, Ethiopia

\section{Email address: \\ eyayafekadie@yahoo.com}

\section{To cite this article:}

Eyaya Fekadie Anley. Numerical Solutions of Elliptic Partial Differential Equations by Using Finite Volume Method. Pure and Applied Mathematics Journal. Vol. 5, No. 4, 2015, pp. 120-129. doi: 10.11648/j.pamj.20160504.16

Received: October 23, 2015; Accepted: November 16, 2015; Published: July 23, 2016

\begin{abstract}
Solution of Partial Differential Equations (PDEs) in some region R of the space of independent variables is a function, which has all the derivatives that appear on the equation, and satisfies the equation everywhere in the region R. Some linear and most nonlinear differential equations are virtually impossible to solve using exact solutions, so it is often possible to find numerical or approximate solutions for such type of problems. Therefore, numerical methods are used to approximate the solution of such type of partial differential equation to the exact solution of partial differential equation. The finite-volume method is a method for representing and evaluating partial differential equations in the form of algebraic equations [LeVeque, 2002; Toro, 1999]. In the finite volume method, volume integrals in a partial differential equation that contain a divergence term are converted to surface integrals, using the divergence theorem. These terms are then evaluated as fluxes at the surfaces of each finite volume. Because the flux entering a given volume is identical to that leaving the adjacent volume, these methods are conservative. Another advantage of the finite volume method is that it is easily formulated to allow for unstructured meshes. The method is used in many computational fluid dynamics packages.
\end{abstract}

Keywords: Finite Volume Method, Discritization, PDEs, Control Volume (CV)

\section{Introduction}

Differential equations are mathematical expressions that how the variables and their derivatives with respect to one or more independent variables affect each other in dynamic way. A partial differential equation is a differential equation in which the unknown function $\mathrm{F}$ is a function of multiple independent variables and of their partial derivatives or Equations involving one or more partial derivatives of a function of two or more independent variables is called partial differential equations (PDEs). The highest derivative in the partial differential equation is the order of the partial differential equation. A PDE is linear if the dependent variable and its functions are all of first order. A PDE is homogeneous if each term in the equation contains either the dependent variable or one of its derivatives. Otherwise, the equation is said to be non-homogeneous in the given partial differential equation. The equation of the form

$$
\begin{gathered}
A(x, y) u_{x x}+B(x, y) u_{x y}+C(x, y) u_{y y}+D(x, y) u_{x} \\
+E(x, y) u_{y}+F(x, y) u=G(x, y)
\end{gathered}
$$

This is the general second order, linear and nonhomogeneous partial differential equation. The partial differential equation is classified as parabolic, hyperbolic and elliptic depending on the values of $\mathrm{A}, \mathrm{B}$ and $\mathrm{C}$ in the above equation (1). That is if the discriminate defined by $\Delta=B^{2}-$ $4 A C>0$, then the above equation (1) is said to be hyperbolic partial differential equation. If the discriminate $\Delta<0$, then the equation is said to be elliptic partial differential equation and if the discriminate $\Delta=0$, then the equation is also said to be parabolic partial differential equation.

PDEs are mathematical models of continuous physical phenomenon in which a dependent variable, say u, is a function of more than one independent variable, say y (time), and $\mathrm{x}$ (eg. spatial position). PDEs derived by applying a physical principle such as conservation of mass, momentum or energy. These equations, governing the kinematic and 
mechanical behavior of general bodies are referred to as Conservation Laws. These laws can be written in either the strong of differential form or an integral form.

\section{Discretization of Partial Differential Equation by Using FVM}

The starting point for a finite-volume discretization is a decomposition of the problem domain $\Omega$ into a finite number of sub domains $v_{i}(i=1,2, \ldots, N)$ called control volumes (CVs), and related nodes where the unknown variables are to be computed. The union of all CVs should cover the whole problem domain. In general, the CVs also may overlap, but since these results in unnecessary complications we consider here the non-overlapping case only. Since finally each CV gives one equation for computing the nodal values, their final number (i.e., after the incorporation of boundary conditions) should be equal to the number of CVs. Usually, the CVs and the nodes are defined on the basis of a numerical grid. For one-dimensional problems the CVs are subintervals of the problem interval and the nodes can be the midpoints or the edges of the sub-intervals.

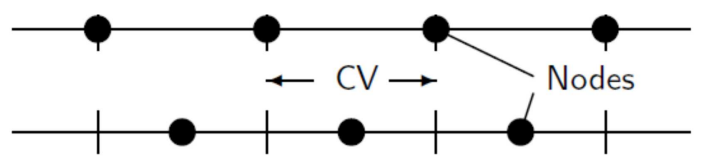

Figure 1. Definitions of CVS and edge (top) and cell-oriented (bottom) arrangement of nodes for One-dimensional grids.

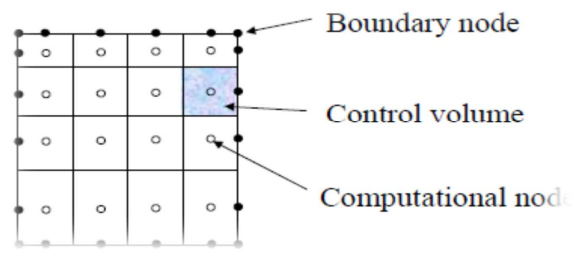

Figure 2. The indication for the place of nodes.

In the two-dimensional case the CVs can be arbitrary polygons. For quadrilateral grids the CVs usually are chosen identically with the grid cells. The nodes can be defined as the vertices or the centers of the CVs often called edge or cell-centered approaches, respectively. For triangular grids, in principle, one could do it similarly, i.e., the triangles define the CVs and the nodes can be the vertices or the centers of the triangles. Here, the nodes are chosen as the vertices of the triangles and the CVs are defined as the polygons formed by the perpendicular bisectors of the sides of the surrounding the triangles.

Definition: Finite Volume Method is a sub-domain method with piecewise definition of the field variable in the neighborhood of chosen control volumes. The total solution domain is divided in to many small control volumes which are usually rectangular in shape. The numerical solution that we are seeking is represented by a discrete set of function values $\left\{u_{1}, u_{2}, u_{3}, \ldots \ldots \ldots \ldots \ldots \ldots \ldots u_{N}\right\}$ that approximate $u$ at these points, i.e $u_{i} \approx u\left(x_{i}\right), i=1,2, \ldots \ldots \ldots \ldots ., N$. In what follows, and unless otherwise stated, we will assume that the points are equally spaced along the domain with a constant distance $\Delta x=x_{i+1}-x_{i}, i=1,2, \ldots \ldots N_{-1}$. This way we will write $u_{i+1} \approx u\left(x_{i+1}\right)=u\left(x_{i}+\Delta x\right)$. This partition of the domain into smaller sub-domains is referred to as a mesh or grid. Using finite volume method, the solution domain is subdivided into a finite number of small control volumes by a grid that grid defines the boundaries of the control volumes while the computational node lies at the center of the control volume.

Nodal points are used within these control volumes for interpolating the field variable and usually, single node at the center of the control volume is used for each control volume.

The finite volume method is a discretization of the governing equation in integral form, in contrast to the finite difference method, which is unusually applied to the governing equation in differential form. In order to obtain a finite volume discretization, the domain $\Omega$ will be Sub divided into $M$ sub-domains $\Omega i$ such that the collection of all those sub domains forms a partition of $\Omega$, that is:

1. Each $\Omega_{i}$ is an open, simply connected, and polygonal bounded set without slits

2. There is no any common point between each sub domains. (i.e. $\Omega_{i} \cap \Omega_{j}=\Phi$ for $i \neq j$

3. The union of all the sub-domain gives the domain of the region. (i.e. $\bigcup^{M} \Omega_{i}=\Omega$ ). These sub domains $\Omega_{i}$ are called control volumes or control domains.

In the cell-centered methods, the unknowns are associated with the control volumes, for example, any control volume corresponds to a function value at some interior point. In the cell-vertex methods, the unknowns are locating at the vertices of the control volumes.
2D

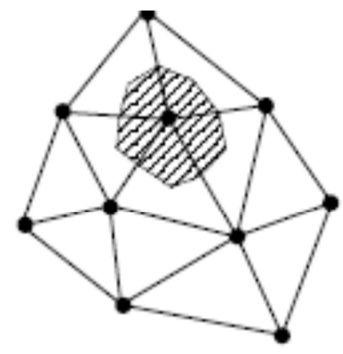

a
2D

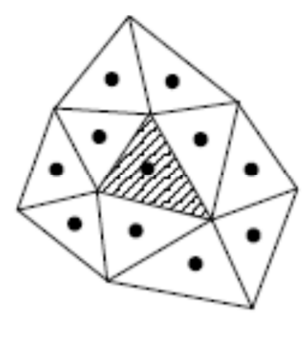

b
Figure 3. a: Vertex-centered FVM, b: Cell centered FVM.

Example 1: Consider the homogeneous Dirichlet problem for the Poisson equation on the unit square

$$
\begin{aligned}
& -u_{x x}(x)=f, \text { in } \Omega=(0,1)^{2} \\
& u(x)=0, \text { on } \partial \Omega
\end{aligned}
$$

The following figure shows that Problem variables and control volumes in a cell-centered finite volume method. Problem variables: Function values at the nodes $a_{i}$ of a 
square grid with mesh width $h>0$.

Control volumes: $\Omega_{i}=\left\{x \in \Omega: \backslash x-a_{i} \backslash_{\infty}<h\right\}$.

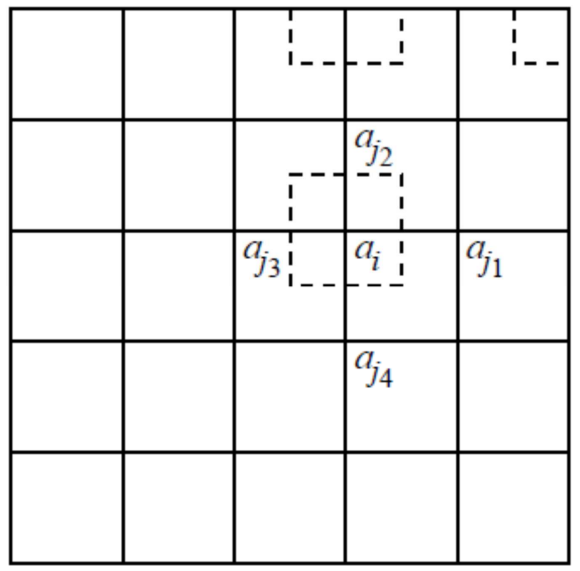

Figure 3. Cell centered FVM.

For an inner control volume $\Omega_{i}\left(i, e . a_{i} \in \Omega\right.$, the above equation becomes as follow. $-\sum_{k=1}^{4} \int_{\Gamma_{i j k}} v_{i j k} u_{x x} d p=\int_{\Omega_{i}} f(x) d x$ Where $\Gamma_{i j k}=\partial \Omega_{i} \cap \partial \Omega_{j k}$. Approximating the integrals on $\Gamma_{i j k}$ by means of the midpoint rule and replacing the derivatives by difference quotients, we have

$$
\begin{aligned}
& -\sum_{k=1}^{4} \int_{\Gamma_{j j k}} v_{i j k} u_{x x} d p \approx-\sum v_{i j k} u_{x x} \frac{\left(a_{i}+a_{j k}\right) h}{2} \approx \\
& -\left[\frac{\left.u\left(a_{j 1}\right)-u\left(a_{i}\right)+u\left(a_{j 2}\right)-u\left(a_{i}\right)-u\left(a_{i}\right)+u\left(a_{j 3}\right)-u\left(a_{i}\right)+u\left(a_{j 4}\right)\right] h}{h}=4 u\left(a_{i}\right)-\sum_{k=1}^{4} u\left(a_{j k}\right)\right.
\end{aligned}
$$

and we can approximate the right hand side by using the midpoint rule. If $a_{i} \in \partial \Omega$, then parts of the boundary $\partial \Omega_{i}$ lie on $\partial \Omega$. At these nodes, the Dirichlet boundary conditions already prescribe values of the unknown function, and so there is no need to include the boundary control volumes into the balance equations.

In order to approximate the value of the solution of partial differential equation by finite volume method we use the following steps.

Step 1: Grid Generation: The first step in the finite volume method is grid generation by dividing the domain in to discrete control volumes. Let us place a number of nodal points in the space between the points. The boundaries of control volumes are positioned mid-way between adjacent nodes. Thus each node is surrounded by a control volume or cell. It is common practice to set up control volumes near the edge of the domain in such a way that the physical boundaries coincide with the control volume boundaries. A general nodal point defined by and its neighbors in a onedimensional geometry.

Step 2: Discretization: The most important features of finite volume method are the integration of the governing equation over a control volume to yield a discretized equation at its nodal points

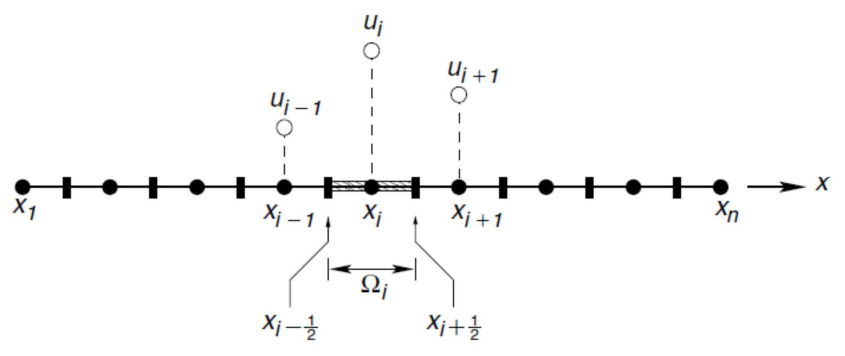

Figure 4. Discretization of the domain.

Step 3: Solution: After discretization over each volume method, we are finding a system of algebraic equation which is easily solved by numerical methods.

\section{Formulation of Finite Volume Scheme of One Dimensional Elliptic PDEs}

The principle of the finite volume method will be shown here on the academic Dirichlet problem, namely a second order differential operator without time dependent terms and with homogeneous Dirichilet boundary conditions. Let $\mathrm{f}$ be a given function from $(0,1)$ to IR, consider the following differential equation: consider the equation of the form $U_{x x}(x)=f(x), x \in(0.1)$ with the boundary condition

$$
u(0)=0, u(1)=0
$$

Let $f \in C([0,1], I R)$ there exists a unique solution $u \in$ $c^{2}([0,1], I R)$ to the Problem (3.1). In the sequel, this exact solution will be denoted by $u$ and (3.1) can be written in the conservative form $\operatorname{div}(\mathrm{F})=\mathrm{f}$, with $\mathrm{F}=u_{x}$. In order to compute a numerical approximation to the solution of this equation, let us define a mesh or grid, denoted by $\mathrm{T}$, of the interval $(0,1)$ consisting of $\mathrm{N}$ cells or control volumes, denoted by $k_{i},=1,2, \ldots \ldots N$ and $\mathrm{N}$ points of $(0,1)$, denoted by $x_{i}, i=12,3 \ldots \ldots . N$, satisfying the following definitions:

Definition: An admissible mesh of $(0,1)$, denoted by $\mathrm{T}$, is given by a family $k_{i}, i=1,2,3, \ldots \ldots . . N, N \in N^{*}$ such that $k_{i}=\left(x_{i-\frac{1}{2}}, x_{i+\frac{1}{2}}\right)$ and a family $\left(x_{i}\right), i=0,1,2, \ldots \ldots . . N_{+1}$ with $\quad x_{0}=0, x_{\frac{1}{2}}<x_{1}<x_{\frac{3}{2}}<, \ldots \ldots x_{i-\frac{1}{2}}<x_{i}<x_{i+\frac{1}{2}}<$ ,.... $x_{N+\frac{1}{2}}=x_{N+1}=1$ and the step size has the following properties $\quad h_{i}=m\left(k_{i}\right)=x_{i+\frac{1}{2}}-x_{i-\frac{1}{2}}, i=1,2,3, \ldots \ldots . . N$ and therefore $\sum_{i=1}^{N} h_{i}=1$ also we have $h_{i}^{-}=x_{i}-x_{i-\frac{1}{2}}$ and $h_{i}^{+}=x_{i+\frac{1}{2}}-x_{i}, i=1,2, \ldots \ldots \ldots . . . N$. Size of the mesh, denoted by, size $(\mathrm{T})=\mathrm{h}=\max \left\{h_{i}, i=1,2,3, N\right)$. The discrete unknowns are denoted $u_{i}, i=1,2,3, \ldots \ldots . N$ and are expected to be some approximation of $\mathrm{u}$ in the cell $k_{i}$ (the discrete unknown $u_{i}$ can be viewed as an approximation of the mean value of $u$ over $k_{i}$ or of the value of $u\left(x_{i}\right)$ or of other 
values of $\mathrm{u}$ in the control volume $k_{i}$. The first equation of (3) is integrated over each cell yield the following.

$$
u_{x}\left(x_{i+\frac{1}{2}}\right)-u_{x}\left(x_{i-\frac{1}{2}}\right)=\int_{k_{i}} f(x) d x .
$$

After integration we get the following expression.

$-u_{x}\left(x_{i+\frac{1}{2}}\right)+u_{x}\left(x_{i-\frac{1}{2}}\right)=\int_{k_{i}} f(x) d x, i=1,2,3$,

The Dirichlet boundary conditions are taken into account by using the values imposed at the boundaries to compute the fluxes on these boundaries. Taking these boundary conditions into consideration and setting $f_{i}=\frac{1}{h_{i}} \int_{k_{i}} f(x) d x$ for $\mathrm{i}=1$, $2 \ldots, \mathrm{N}$ in an actual computation, an approximation of $f_{i}$ by numerical integration of mid-point rule can be used.

The finite volume scheme for the problem (3) can be written as follows,

$$
F_{i+\frac{1}{2}}-F_{i-\frac{1}{2}}=h_{i} f_{i}, \text { For } \mathrm{i}=1,2, \ldots, \mathrm{N} .
$$

Where

$$
\begin{gathered}
F_{i-\frac{1}{2}=\frac{u_{i-u_{i-1}}}{h_{i-\frac{1}{2}}} \text { for } \mathrm{i}}=1,2, \ldots, \mathrm{N}- \\
F_{\frac{1}{2}}=\frac{-u_{1}}{h_{\frac{1}{2}}} \\
F_{N+\frac{1}{2}}=\frac{u_{N}}{h_{N+\frac{1}{2}}}
\end{gathered}
$$

The equation in (6) -(8) can be written as

$$
F_{i+\frac{1}{2}}=\frac{u_{i+1}-u_{i}}{h_{i+\frac{1}{2}}} \text { for } \mathrm{i}=0,1,2, \ldots, \mathrm{N}
$$

By setting

$$
u_{0}=u_{N+1}=0
$$

The numerical scheme (5)-(8) may be written under the following matrix form:

$$
\mathrm{AU}=\mathrm{b},
$$

Where $U=\left(u_{1}, \ldots, u_{N}\right)^{t}, b=\left(b 1, \ldots, b_{N}\right)^{t}$, with (10) and with $A$ and $b$ defined by

$(A U)_{i}=\frac{1}{h_{i}}\left(\frac{u_{i+1}-u_{i}}{h_{i+\frac{1}{2}}}-\frac{u_{i}-u_{i-1}}{h_{i-\frac{1}{2}}}\right), i=1,2,3, \ldots . . N$. whereb $_{i}=\frac{1}{h_{i}}$

Example 2: Consider the second order one-dimensional linear elliptic problem:

$u_{x x}(x)=-\sin x, x \in(0,1)$, with boundary conditions: $u(0)=0, u(1)=0.017452406$

The exact solution of the problem is $u(x)=\sin x$
Solution Now to solve the above problem, integrating equation (3) over each control volume $\left(x_{i+\frac{1}{2}}, x_{i-\frac{1}{2}}\right)$ and considering $h=0.05, \mathrm{i}=1,2,3, \ldots, 19$. The finite scheme related to (3) is given by $20 u_{i+1}-40 u_{i}+20 u_{i-1}$ $=\cos \left(x_{i+\frac{1}{2}}\right)-\cos \left(x_{i-\frac{1}{2}}\right), i=1,2, \ldots ., 19$.

Table 1. Numerical results for example (2) by using FVM.

\begin{tabular}{lllll}
\hline $\mathbf{i}$ & $\boldsymbol{x}_{\boldsymbol{i}}$ & $\boldsymbol{u}_{\boldsymbol{i}}$ & Exact solution & Absolute error \\
\hline 0 & 0 & 0 & 0 & 0 \\
1 & 0.05 & 0.0009 & 0.000872664 & 0.000027336 \\
2 & 0.1 & 0.0018 & 0.001745328 & 0.000054672 \\
$\ldots$ & $\ldots$ & $\ldots$ & $\ldots$ & $\ldots$ \\
20 & 1.0 & 0.017452406 & 0.017452406 & 0 \\
\hline
\end{tabular}

Theorem 1: Let $f \in C([0,1], I R)$ and let $u \in$ $c^{2}([0,1], I R)$ be the unique solution of Problem (3). Let $T=$ $(K i) i=1, \ldots, N$ be an admissible mesh in the case of the above definition. Then, there exists a unique vector $\mathrm{U}=$ $\left(u_{1, \ldots \ldots \ldots . . .,} u_{N}\right)^{t} \in I R^{N}$ be the solution to (5) - (8) and there exists $C \geq 0$, only depending on $u$, such that,

$$
\begin{gathered}
\sum_{i=0}^{N} \frac{\left(e_{i+1 \_} e_{i}\right)^{2}}{h_{i+\frac{1}{2}}} \leq C^{2} h^{2}, \\
\text { And } e_{i} \leq C h, \forall_{i}(1,2,3, \ldots \ldots \ldots, N)
\end{gathered}
$$

with $e_{0=} e_{N+1}=0$ and $e_{i}=u\left(x_{i}\right)-u_{i}$

Table 2. Error estimate of one-dimensional elliptic problem by using theorem 1 of Table 1.

\begin{tabular}{lll}
\hline $\mathbf{I}$ & $\frac{\left(\boldsymbol{e}_{i+1}-\boldsymbol{e}_{\boldsymbol{i}}{ }^{2}\right.}{\boldsymbol{h}_{\boldsymbol{i}+\frac{1}{2}}}$ & $\mathbf{a b s}\left(\boldsymbol{e}_{\boldsymbol{i}}\right)$ \\
\hline 0 & 0.000000014 & 0 \\
1 & 0.000000014 & 0.000027336 \\
2 & 0.000000026 & 0.000054672 \\
$\ldots$ & $\ldots$ & $\ldots$ \\
19 & 0.000000008 & 0.000020132 \\
\hline
\end{tabular}

Now to estimate the error using the equation (13) implies $\mathrm{c}=0.1$

Then $\sum_{i=0}^{19} \frac{\left(e_{i+1}-e_{i}\right) 2}{h_{i+\frac{1}{2}}} \leq 0.000025$ and $a b\left(e_{i}\right) \leq 0.005, i=$ $1,2, \ldots, N$

\section{Finite Volume Method for Two Dimensions of Elliptic PDEs}

\subsection{Formulation of Finite Volume Method for Linear Systems of PDEs}

This section is concerned with the discretization of linear system of two dimensions of elliptic partial differential equation by finite volume method (FVM) on $\Omega=(0, a) \times$ $(0, a)$ with rectangular meshed and let $\Omega$ be an open bounded 
polygonal subset of $\mathrm{IR}^{2}$ and admissible finite volume mesh of $\Omega$, denoted, by $\mathrm{T}$ is given by a family of control volumes which are open polygonal convex subset of $\Omega$.

Consider the partial differential equation of the form

$$
u_{x x}(x, y)+u_{y y}(x, y)=f(x, y),(x, y) \in \Omega
$$

$u(x, y)=0$ where $(x, y)$ is included in the boundary of the domain

Let $\Omega=(0, \mathrm{a}) \times(0, \mathrm{a}) \operatorname{andf}_{1} \mathrm{f}_{2}, \mathrm{C}^{2}(\Omega, \mathrm{R})$ and let $T=\left(k_{i, j}\right) i=1,2, \ldots, N_{1} ; j=1,2, \ldots, N_{2}$; be an admissible mesh of $(0, a) \times(0, a)$ that satisfying the following additional assumption.

$h_{1}, h_{2}, \ldots \ldots \ldots \ldots \ldots h_{N_{1}}>0, k_{1}, k_{2}, \ldots \ldots \ldots . . k_{N_{2}}>0$, Such that $\sum_{i=1}^{N_{1}} h_{i}=1, \sum_{j=1}^{N_{2}} k_{j}=1$ and let

$h_{0}=0, h_{N_{1}+1}=0$ and $k_{0}=0, k_{N_{2}+1}=0$. For $i=1,2, \ldots \ldots \ldots . . N_{1}$. Let $x_{\frac{1}{2}}=0$ and

$x_{i+\frac{1}{2}}=x_{i-\frac{1}{2}}+h_{i}$, so that $x_{N_{1+\frac{1}{2}}}=1$ and for $\mathrm{j}=1$,

$2, \ldots \ldots, \mathrm{N}_{2}$

$\mathrm{y}_{\frac{1}{2}}=0, \mathrm{y}_{\mathrm{j}+\frac{1}{2}}=\mathrm{y}_{\mathrm{j}-\frac{1}{2}}+\mathrm{k}_{\mathrm{j}}$, so that $\mathrm{y}_{\mathrm{N} 2+\frac{1}{2}}=1$ and $\mathrm{k}_{\mathrm{i}, \mathrm{j}}=$ $\left[\mathrm{x}_{\mathrm{i}-\frac{1}{2}}, \mathrm{x}_{\mathrm{i}+\frac{1}{2}}\right] \times\left[\mathrm{y}_{\mathrm{j}-\frac{1}{2}}, \mathrm{y}_{\mathrm{j}+\frac{1}{2}}\right]$

Let with, $\left\{\mathrm{x}_{\mathrm{i}}\right\}, \mathrm{i}=0,1,2, \mathrm{~N}_{1+} 1\left\{\mathrm{y}_{\mathrm{j}}\right\}, \mathrm{j}=0,1,2, \ldots \ldots, \mathrm{N}_{2}+$ 1 such that $\mathrm{x}_{\mathrm{i}-\frac{1}{2}}<\mathrm{x}_{\mathrm{i}}<\mathrm{x}_{\mathrm{i}+\frac{1}{2}}$ for

$\mathrm{i}=1,2, \ldots \ldots, N_{1}+1$ and $x_{0}=0, x_{N_{1}+1}=1$.Similarlly $y_{j-\frac{1}{2}}<y_{j}<y_{j+\frac{1}{2}}$ for

$$
\int_{y-\frac{1}{2}}^{y}{ }_{j+\frac{1}{2}}\left(u_{x}\left(x_{i+\frac{1}{2}}, y\right)-u_{x}\left(x_{i-\frac{1}{2}}, y\right)\right) d y+\int\left(u_{y}\left(x, y_{j+\frac{1}{2}}\right)-u_{y}\left(x, y_{j-\frac{1}{2}}\right)\right) d x-0.04 \sin u_{i, j}=-0.004
$$

And by using finite difference a method, we have

$$
\frac{k_{j}}{h_{i+\frac{1}{2}}}\left(u_{i+1, j}-u_{i, j}\right)-\frac{k_{j}}{h_{i-\frac{1}{2}}}\left(u_{i, j}-u_{i-1, j}\right)+\frac{h_{i}}{k_{j+\frac{1}{2}}}\left(u_{i, j+1}-u_{i, j}\right)-\frac{h_{i}}{k_{j-\frac{1}{2}}}\left(u_{i, j}-u_{i, j-1}\right)-0.04 \sin u_{i, j}=-0.004
$$

By using mid- point rule and FDM, the finite volume scheme gives the following.

$$
\begin{gathered}
u_{i+1, j}-4 u_{i, j}+u_{i-1, j}+u_{i, j+1}+u_{i, j-1}-0.04 \sin u_{i, j} \\
=0.004
\end{gathered}
$$

$i=1,2, N_{2}+1 \quad$ and $\quad y_{0}=0, y_{N_{2}+1}=1 \quad$ and let $x_{i, j}=\left(x_{i}, y_{j}\right)$ for $i=1,2, \ldots \ldots \ldots \ldots, N_{1}, j=1,2, N_{2}$.

Set $h_{i}^{-}=x_{i}-x_{i-\frac{1}{2}}, h_{i}^{+}=x_{i+\frac{1}{2}}-x_{i}$ for $i=1,2, N_{1}$ with $h_{i+\frac{1}{2}}=x_{i+1}-x_{i}$

$\forall_{i} \in\left(0,1,2, \ldots, N_{1}\right)$. Similarly, $k_{j}^{-}=y_{j}-y_{j-\frac{1}{2}}, k_{j}^{+}=$ $y_{j+\frac{1}{2}}-k_{j}, \mathrm{j}=1,2 \ldots ., \mathrm{N}_{2}, k_{j+\frac{1}{2}}=y_{j+1}-y_{j}$ for $\mathrm{j}=0,1$,

$\mathrm{N}_{2} \cdot h=\max \left\{\left(h_{i}, i=1,2,3, \ldots \ldots \ldots \ldots \ldots . . . N_{1}\right),\left(k_{j} j=1,2, \ldots \ldots . . N_{2}\right)\right\}$

Theorem 2: Let $\Omega=\left(x_{i-\frac{1}{2}}, x_{i+\frac{1}{2}}\right) \times\left(y_{j-\frac{1}{2}}, y_{j+\frac{1}{2}}\right)$ be the domain and $\mathrm{f} \in C^{2}(\Omega)$, let $\mathrm{u}$ be th unique variational solution of elliptic partial differential equation. Let $\varepsilon>0$ be such that

$h_{i}>$ ch for $i=1,2,3, ., N_{2}$. Then there exists unique solution $u_{i j}, i=1,2, \ldots \ldots \ldots \ldots N_{1}, j=1,2, \ldots \ldots \ldots \ldots . . N_{2}$ such that the formula $F_{i+\frac{1}{2}, j}+F_{i-\frac{1}{2}, j}+F_{i, j+\frac{1}{2}}+F_{i, j-\frac{1}{2}}=h_{i, j} f_{i, j}$ holds.

Example 3: Consider the boundary value problem of elliptic problem of Sine-Gordon's equation

$$
\begin{gathered}
u_{x x}(x, y)+u_{y y}(x, y)-\sin u(x, y)=-\sin x \\
0 \leq x \leq 1,0 \leq y \leq 1 \\
u(x, 0)=x, u(x, 1)=x, 0 \leq x \leq 1 \\
u(0, y)=0, u(1, y)=1,0 \leq y \leq 1
\end{gathered}
$$

The exact solution of the problem is $u(x, y)=x$. with $\mathrm{h}=$ $\mathrm{k}=0.2$, after integrating over the control volumes $k_{i, j}=$ $\left(x_{i-\frac{1}{2}}, x_{i+\frac{1}{2}}\right) \times\left(y_{j-\frac{1}{2}}, y_{j+\frac{1}{2}}\right)$ one can get the following.

Table 3. Comparison between the numerical and exact solutions of the example 3 by using FVM with $k^{0}=1$.

\begin{tabular}{llllllll}
\hline $\mathbf{I}$ & $\boldsymbol{x}_{\boldsymbol{i}}$ & $\boldsymbol{u}_{\boldsymbol{i}, \mathbf{1}}^{(\mathbf{1})}$ & $\boldsymbol{u}_{\boldsymbol{i , \mathbf { 2 }}}^{(\mathbf{1})}$ & $\boldsymbol{u}_{\boldsymbol{i}, \mathbf{3}}^{(\mathbf{1})}$ & $\boldsymbol{u}_{\boldsymbol{i , \mathbf { 4 }}}^{(\mathbf{1})}$ & Exact solution & $\operatorname{Abs}\left(E . S-\boldsymbol{u}_{\boldsymbol{i , \mathbf { 4 }}}^{(\mathbf{1})}\right)$ \\
\hline 0 & 0 & 0 & 0 & 0 & 0 & 0 & 0 \\
1 & 0.2 & 0.2238 & 0.2326 & 0.2326 & 0.2238 & 0.2000 & 0.0238 \\
$\ldots$ & $\ldots$ & $\ldots$ & $\ldots$ & $\ldots$ & $\ldots$ & $\ldots$ & $\ldots$ \\
5 & 1 & 1.0000 & 1.0000 & 1.0000 & 1.0000 & 1.0000 & 0 \\
\hline
\end{tabular}


and the following solution is obtained for $k^{0}=2$ :

Table 4. Comparison between the numerical and exact solutions of the example 3 by using $F V M$ with $k^{0}=2$.

\begin{tabular}{|c|c|c|c|c|c|c|c|}
\hline I & $x_{i}$ & $u_{i, 1}^{(2)}$ & $u_{i, 2}^{(2)}$ & $u_{i, 3}^{(2)}$ & $u_{i, 4}^{(2)}$ & Exact solution & $\operatorname{Abs}\left(\operatorname{Exact}-u_{i, 4}^{(2)}\right)$ \\
\hline 0 & 0 & 0 & 0 & 0 & 0 & 0 & 0 \\
\hline 1 & 0.2 & 0.2039 & 0.2055 & 0.2055 & 0.2039 & 0.2000 & 0.0039 \\
\hline$\ldots$ & $\ldots$ & $\ldots$ & $\ldots$ & $\ldots$ & $\ldots$ & $\ldots$ & $\ldots$ \\
\hline 5 & 1 & 1.0000 & 1.0000 & 1.0000 & 1.0000 & 1.0000 & 0 \\
\hline
\end{tabular}

Also the following solution is obtained $k^{0}=3$

Table 5. Comparison between the numerical and exact solutions of the example 3 by using $F V M$ with $k^{0}=3$.

\begin{tabular}{|c|c|c|c|c|c|c|c|}
\hline I & $x_{i}$ & $u_{i, 1}^{(3)}$ & $u_{i, 2}^{(3)}$ & $u_{i, 3}^{(3)}$ & $u_{i, 4}^{(3)}$ & Exact solution & $\operatorname{Abs}\left(\operatorname{Exact}-u_{i, 4}^{(3)}\right)$ \\
\hline 0 & 0 & 0 & 0 & 0 & 0 & 0 & 0 \\
\hline 1 & 0.2 & 0.2032 & 0.2044 & 0.2044 & 0.2032 & 0.2000 & 0.0032 \\
\hline$\ldots$ & $\ldots$ & $\ldots$ & $\ldots$ & $\ldots$ & $\ldots$ & $\cdots$ & $\cdots$ \\
\hline 5 & 1 & 1.0000 & 1.0000 & 1.0000 & 1.0000 & 1.0000 & 0 \\
\hline
\end{tabular}

Now using the data that are given in the three tables, we can draw the figure on the same coordinate axes as follow

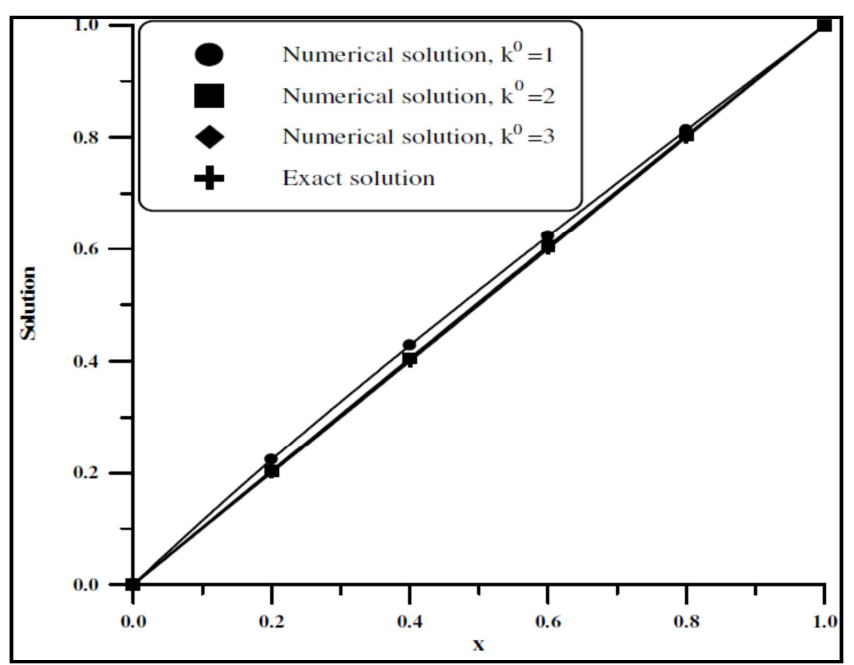

Figure 5. The numerical and exact solutions of example (3) by using FVM.

\subsection{The Formulation of Finite Volume Method of \\ Nonlinear System of Two Dimensions of Elliptic Partial Differential Problems}

Since the finite volume method (FVM) for nonlinear twodimensional elliptic problem of PDE is difficult to be used directly; thus the Newton's method used to overcome such computational difficulties. This method is used to find the nonlinear partial differential equation. In these case the functional iteration is written as:

$$
W^{\left(K^{0}\right)}=W^{\left(K^{0}-1\right)}-J\left(\mathrm{~W}^{\left(\mathrm{K}^{0}-1\right)^{-1}}\right) F\left(W^{\left(K^{0}-1\right)}\right)
$$

Where

$$
F(W)=\left(f_{1}\left(u_{11}, u_{21}, \ldots, u_{n n}, v_{11}, v_{21}, \ldots, v_{n n}\right),\right.
$$

$$
\begin{gathered}
f_{2}\left(u_{11}, u_{21}, \ldots, u_{n n}, v_{11}, v_{21}, \ldots, v_{n n}\right), \ldots, \\
\left.f_{n}\left(u_{11}, u_{21}, \ldots, u_{n n}, v_{11}, v_{21}, \ldots, v_{n n}\right)\right) t \\
W^{\left(K^{0}\right)}=\left(u_{11}^{\left(K^{0}\right)}, u_{21}^{k^{0}}, \ldots u_{n n}^{k^{0}}, v_{11}^{k^{0}}, v_{21}^{k^{0}}, \ldots, v_{n n}^{k^{0}}\right)^{t}
\end{gathered}
$$

Where

$$
\begin{aligned}
& \left\lceil\frac{\partial \mathrm{f}_{1}}{\partial \mathrm{u}_{11}}(\mathrm{x}, \mathrm{y}) \frac{\partial \mathrm{f}_{1}}{\partial \mathrm{u}_{21}}(\mathrm{x}, \mathrm{y}) \cdots \frac{\partial \mathrm{f}_{1}}{\partial \mathrm{u}_{\mathrm{nn}}}(\mathrm{x}, \mathrm{y}) \frac{\partial \mathrm{f}_{1}}{\partial \mathrm{v}_{11}}(\mathrm{x}, \mathrm{y}) \frac{\partial \mathrm{f}_{1}}{\partial \mathrm{v}_{21}}(\mathrm{x}, \mathrm{y}) \cdots \frac{\partial \mathrm{f}_{1}}{\partial \mathrm{v}_{\mathrm{nn}}}(\mathrm{x}, \mathrm{y})\right. \\
& J(W)=\frac{\partial f_{2}}{\partial u_{11}}(x, y) \quad \frac{\partial f_{2}}{\partial u_{21}}(x, y) \quad \cdots \frac{\partial f_{2}}{\partial u_{n n}}(x, y) \frac{\partial f_{2}}{\partial v_{11}}(x, y) \frac{\partial f_{2}}{\partial v_{21}}(x, y) \quad \cdots \frac{\partial f_{2}}{\partial v_{n n}}(x, y) \\
& \frac{\partial \mathrm{f}_{\mathrm{n}}}{\partial \mathrm{u}_{11}}(\mathrm{x}, \mathrm{y}) \frac{\partial \mathrm{f}_{\mathrm{n}}}{\partial \mathrm{u}_{21}}(\mathrm{x}, \mathrm{y}) \cdots \frac{\partial \mathrm{f}_{\mathrm{n}}}{\partial \mathrm{u}_{\mathrm{nn}}}(\mathrm{x}, \mathrm{y}) \frac{\partial \mathrm{f}_{\mathrm{n}}}{\partial \mathrm{v}_{11}}(\mathrm{x}, \mathrm{y}) \frac{\partial \mathrm{f}_{\mathrm{n}}}{\partial \mathrm{v}_{21}}(\mathrm{x}, \mathrm{y}) \cdots \frac{\partial \mathrm{f}_{\mathrm{n}}}{\partial \mathrm{v}_{\mathrm{nn}}}(\mathrm{x}, \mathrm{y})
\end{aligned}
$$

Where $\mathrm{J}(\mathrm{W})$ is the $n \times n$ Jacobian matrix.

Example 4: Consider the boundary value problem of twodimensional elliptic problem:

$$
\begin{gathered}
u_{x x}(x, y)+u_{y y}(x, y)-u(x, y) v(x, y) \\
=-x^{2} y^{2}\left(x^{2}+y^{2}\right)+4, x \in(0,1), y \in(0,1) \\
v_{x x}(x, y)+v_{y y}(x, y)-u^{2}(x, y) \\
=-\left(x^{2}+y^{2}\right)^{2}+2\left(x^{2}+y^{2}\right), x \in(0,1), y \in(0,1)
\end{gathered}
$$

with boundary conditions

$$
\begin{gathered}
u(0, y)=x^{2}, u(1, y)=1+y^{2}, 0 \leq y \leq 1 \\
v(0, y)=0, v(1, y)=y^{2}, \mathrm{u}(\mathrm{x}, 0)=\mathrm{x}^{2}, \mathrm{u}(\mathrm{x}, 1) \\
=1+\mathrm{x}^{2}, 0 \leq \mathrm{x} \leq 1 \\
v(x, 0)=0, v(x, 1)=x^{2}
\end{gathered}
$$

The exact solution of the problem is $u(x, y)=x^{2}+$ $y^{2}, v(x, y)=x^{2} y^{2}$.

With $h=0.2, k=0.2$, we have:

Now, integrating equations (18) and (19) over each control volume.

The results of the above integral are given by: 


$$
\begin{gathered}
\frac{k_{j}}{h}\left(u_{i+1, j}-u_{i, j}\right)-\frac{k_{j}}{h_{i+\frac{1}{2}}}\left(u_{i, j}-u_{i-1, j}\right)+\frac{h_{i}}{k_{j+\frac{1}{2}}}\left(u_{i, j+1}-u_{i, j}\right)-\frac{h_{i}}{k_{j-\frac{1}{2}}}\left(u_{i, j}-u_{i, j-1}\right)-0.04 u_{i, j} v_{i, j}=0.1545 \\
\frac{k_{j}}{h_{i+\frac{1}{2}}}\left(v_{i+1, j}-v_{i, j}\right)-\frac{k_{j}}{h_{i-\frac{1}{2}}}\left(v_{i, j}-v_{i-1, j}\right)+\frac{h_{i}}{k_{j+\frac{1}{2}}}\left(v_{i, j+1}-v_{i, j}\right)-\frac{h_{i}}{k_{j-\frac{1}{2}}}\left(v_{i, j}-v_{i, j-1}\right)-0.04 u_{i, j}^{2}=0.0105
\end{gathered}
$$

After some computations, we have the following expression.

$$
\begin{gathered}
u_{i+1, j}-4 u_{i, j}+u_{i-1, j}+u_{i, j+1}+u_{i, j-1}-0,04 u_{i, j} v_{i, j}=0.1545 \\
v_{i+1, j}-4 v_{i, j}+v_{i-1, j}+v_{i, j+1}+v_{i, j-1}-0.04 u_{i, j}^{2}=0.0105
\end{gathered}
$$

We can write the above equation in terms of iteration as follow.

$$
\begin{gathered}
u_{i+1, j}^{k^{0}}-4 u_{i, j}^{k^{0}}+u_{i-1, j}^{k^{0}}+u_{i, j+1}^{k^{0}}+u_{i, j-1}^{k^{0}}-0.04 u_{i, j} v_{i, j}=0.1545 \\
u_{i+1, j}^{k^{0}}-4 u_{i, j}^{k^{0}}+u_{i-1, j}^{k^{0}}+u_{i, j+1}^{k^{0}}+u_{i, j-1}^{k^{0}}-0.04 u_{i, j}^{2}=0.0105
\end{gathered}
$$

Moreover we have the following values of $u$.

$$
\begin{gathered}
u_{0,0}=0, u_{0,1}=0.04, u_{0,2}=0.16, u_{0,3}=0.36, u_{0,4}=0.64, u_{0,5}=1 \\
u_{1,0}=0.04, u_{2,0}=0.16, u_{3,0}=0.36, u_{4,0}=0.64, u_{5,0}=1
\end{gathered}
$$

\begin{tabular}{|c|c|c|c|c|c|c|c|}
\hline I & $x_{i}$ & $v_{i, 1}^{(1)}$ & $v_{i, 1}^{(1)}$ & $v_{i, 1}^{(1)}$ & $v_{i, 1}^{(1)}$ & Exact-solution & $\operatorname{Abs}\left(\operatorname{exact}-v_{i, 4}^{(1)}\right)$ \\
\hline 0 & 0 & 0 & 0 & 0 & 0 & 0 & 0 \\
\hline 1 & 0.2 & 0.0244 & 0.0384 & 0.0466 & 0.0489 & 0.0256 & 0.0233 \\
\hline$\ldots$ & $\ldots$ & $\ldots$ & $\ldots$ & $\ldots$ & $\ldots$ & $\ldots$ & $\ldots$ \\
\hline 5 & 1 & 0.04 & 0.16 & 0.36 & 0.64 & 0.64 & 0 \\
\hline
\end{tabular}

Also the values of $v$ are evaluated as follows.

\begin{tabular}{|c|c|c|c|c|c|c|c|}
\hline I & $x_{i}$ & $u_{i, 1}^{(2)}$ & $u_{i, 2}^{(2)}$ & $u_{i, 3}^{(2)}$ & $u_{i, 4}^{(2)}$ & Exact solution & $\operatorname{Abs}\left(\operatorname{exact}-u_{i, 4}^{(2)}\right)$ \\
\hline 0 & 0 & 0 & 0 & 0 & 0 & 0 & 0 \\
\hline 1 & 0.2 & 0.0839 & 0.1917 & 0.3935 & 0.6772 & 0.6800 & 0.0028 \\
\hline ... & $\ldots$ & $\ldots$ & $\ldots$ & $\ldots$ & $\ldots$ & $\ldots$ & $\ldots$ \\
\hline 5 & 1 & 1.04 & 1.16 & 1.36 & 1.64 & 1.64 & 0 \\
\hline
\end{tabular}

$$
v_{0,0}=v_{0,1}=v_{0,2}=v_{0,3}=v_{0,4}=v_{0,5}=0 \text { and } v_{1.0}=v_{2,0}=v_{3,0}=v_{4,0}=v_{5,0}=0
$$

The following results are obtained.

Table 6. Numerical results for example (4) by using FVM when $k^{0}=1$.

\begin{tabular}{llllllll}
\hline $\mathbf{I}$ & $\boldsymbol{x}_{\boldsymbol{i}}$ & $\boldsymbol{u}_{\mathbf{i}, \mathbf{1}}^{\mathbf{1}}$ & $\boldsymbol{u}_{\mathbf{i}, \mathbf{2}}^{\mathbf{1}}$ & $\boldsymbol{u}_{\mathbf{i}, \mathbf{3}}^{\mathbf{1}}$ & $\boldsymbol{u}_{\mathbf{i}, \mathbf{4}}^{\mathbf{1}}$ & Exact-solution & Abs(exact- $\left.\boldsymbol{u}_{\boldsymbol{i}, \mathbf{4}}^{\mathbf{1}}\right)$ \\
\hline 0 & 0 & 0 & 0 & 0 & 0 & 0 & \\
1 & 0.2 & 0.1053 & 0.2304 & 0.4249 & 0.6937 & 0.6800 & \\
$\ldots$ & $\ldots$ & $\ldots$ & $\ldots$ & $\ldots$ & $\ldots$ & $\ldots$ & $\ldots$ \\
5 & 1 & 1.04 & 1.16 & 1.36 & 1.64 & 1.64 & $\ldots$ \\
\hline
\end{tabular}

Table 7. Comparison between the numerical and exact solutions of example (4) by using FVM when $k^{0}=1$.

Table 8. Numerical results for example (4) by using FVM when $k^{0}=2$.

Table 9. Comparison between the numerical and exact solutions of example 4 by using FVM when $k^{0}=2$.

\begin{tabular}{llllllll}
\hline $\mathbf{I}$ & $\boldsymbol{x}_{\boldsymbol{i}}$ & $\boldsymbol{v}_{\boldsymbol{i}, \mathbf{i}}^{(2)}$ & $\boldsymbol{v}_{\boldsymbol{i}, \mathbf{2}}^{(2)}$ & $\boldsymbol{v}_{\boldsymbol{i}, \mathbf{3}}^{(\mathbf{2})}$ & $\boldsymbol{v}_{\boldsymbol{i}, \mathbf{4}}^{(2)}$ & Exact solution \\
\hline 0 & 0 & 0 & 0 & 0 & 0 & 0 & 0 \\
1 & 0.2 & 0.0079 & 0.0210 & 0.0350 & 0.0441 & 0.0256 & 0.0185 \\
$\ldots$ & $\ldots$ & $\ldots$ & $\ldots$ & $\ldots$ & $\ldots$ & $\ldots$ & 0.64 \\
5 & 1 & 0.04 & 0.16 & 0.36 & 0.64 & 0 \\
\hline
\end{tabular}


Using the above set of data we can draw the figure of exact versus numerical solution.

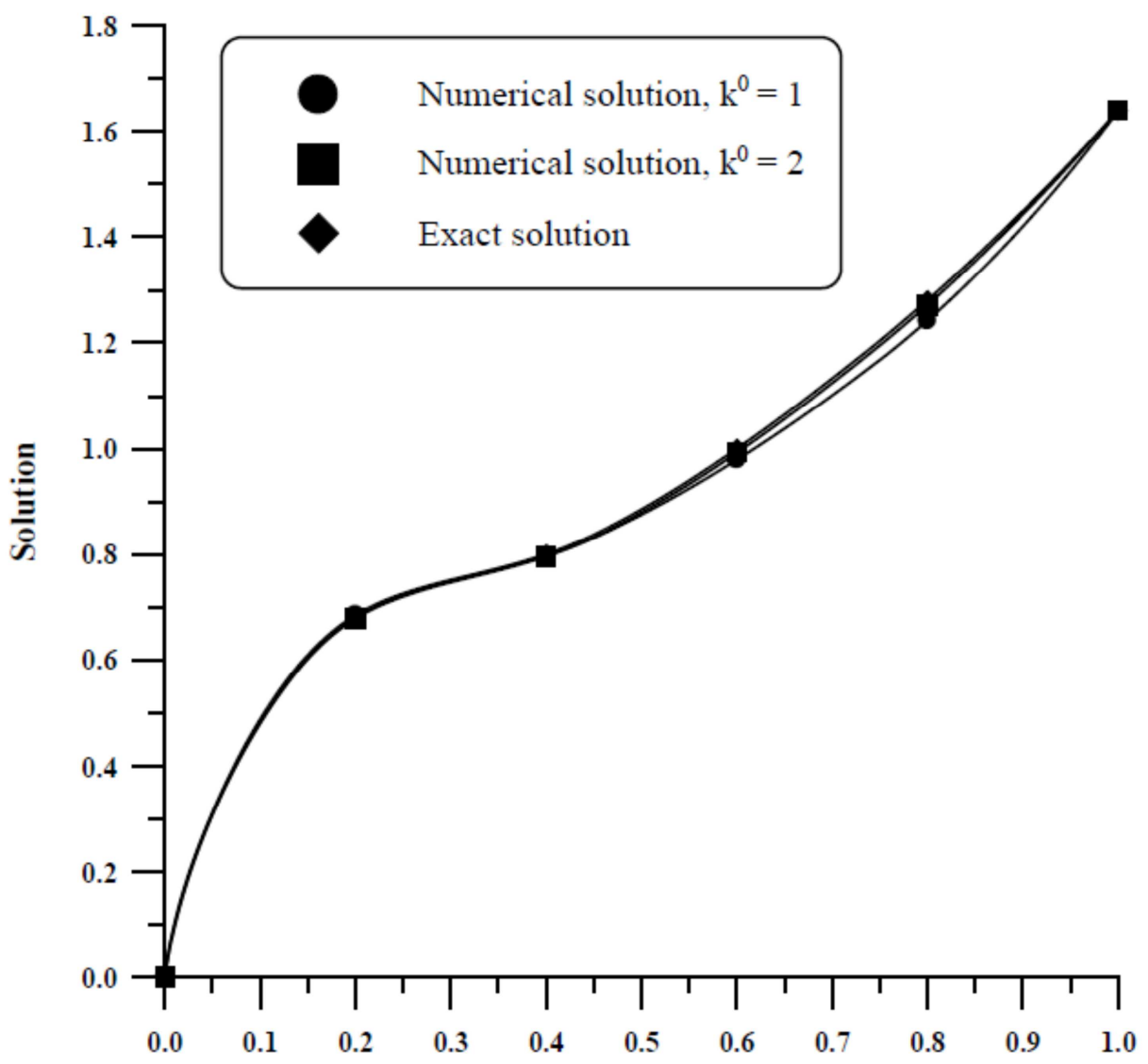

Figure 6. Numerical and exact results for $u, k=1,2$ of example (4) by using FVM.

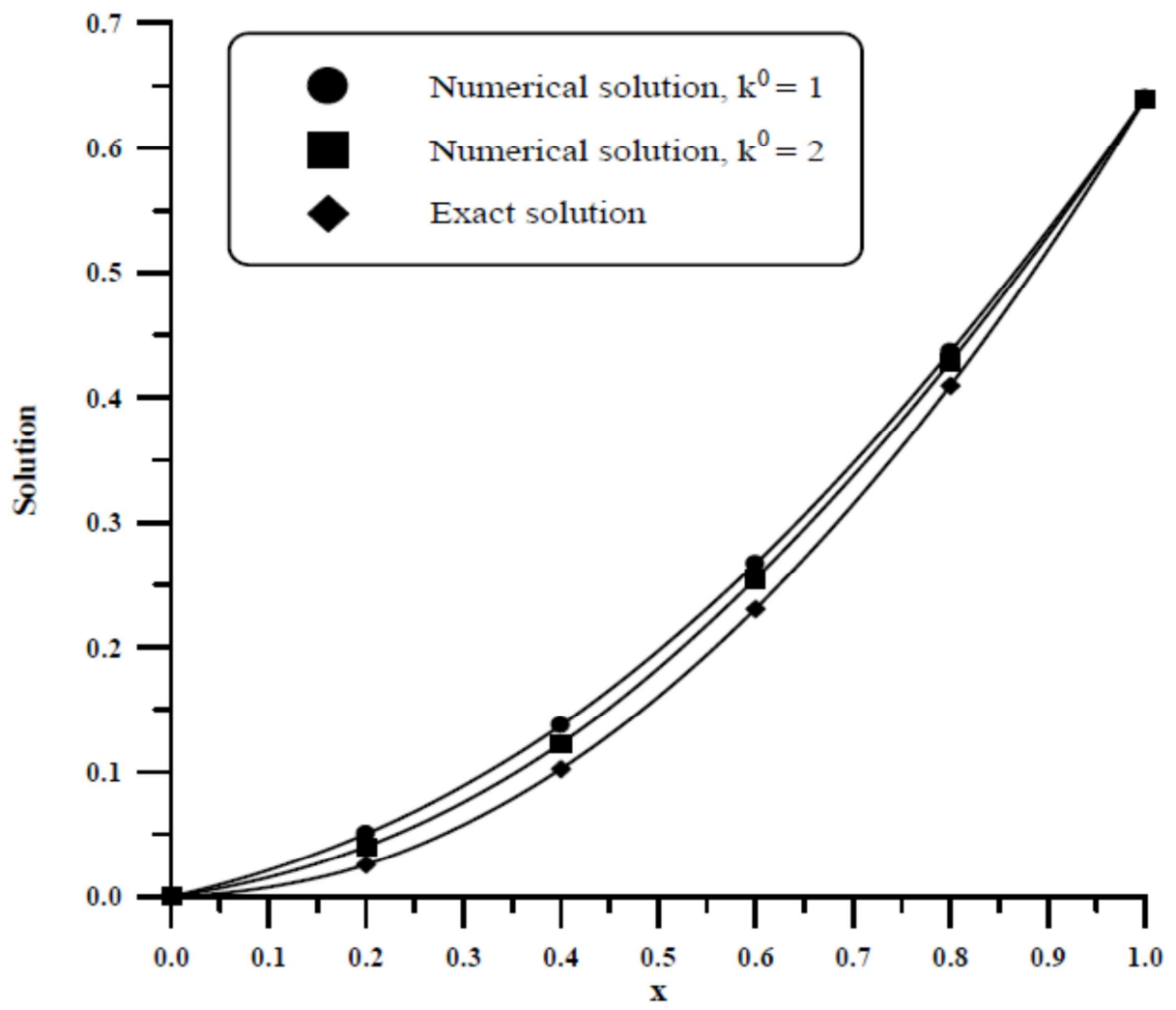

Figure 7. Numerical and exact results for v, $k=1,2$ of example (4) by using FVM. 


\subsection{Error Estimates of Two Dimensional Elliptic Problem of Dirichlet Boundary Conditions}

Theorem 3: Let $\Omega=(0,1) \times(0,1)$ be the domain and $f \in c^{2}(\Omega)$. Let $\mathrm{u}$ be the unique variation solution to equation (17). Under the assumptions of equation (18). Let $\varepsilon>0$ be such that $h_{i}>\varepsilon h, i=1,2, \ldots \ldots . N_{1}, K_{j}>\varepsilon h, j=1.2 \ldots \ldots \ldots . N_{2}$. Then, there exists a unique solution $u_{i, j}, i=1,2, \ldots \ldots N_{1}$ and $j=1,2, \ldots \ldots N_{2}$ Moreover, there exists $\mathrm{C}>0$ only depending on $\mathrm{u}, \Omega$ and $\varepsilon$ such that we have,

$$
\sum_{i, j} \frac{\left(e_{i+1, j}-e_{i, j}\right)^{2}}{h_{i+\frac{1}{2}}} k_{j}+\sum_{i, j} \frac{\left(e_{i, j+1}-e_{i, j}\right)^{2}}{k_{j+\frac{1}{2}}} h_{i} \leq C h^{2}
$$

And

$$
\sum_{i, j}\left(e_{i, j}\right)^{2} h_{i} k_{j} \leq C h^{2}
$$

Where $e_{i, j}=u\left(x_{i, j}\right)-u_{i, j}$, for $i=1,2, N_{1}, j=1,2, \ldots, N_{2}$.

The application of the above theorem to estimate the error of two-dimensional elliptic problem followed in example 2.

\begin{tabular}{|c|c|c|c|c|c|}
\hline i & $x_{i}$ & $\frac{\left(e_{i+1,1}-e_{i, 1}\right)^{2}}{h_{i+\frac{1}{2}}} k_{1}$ & $\frac{\left(e_{i+1,2}-e_{i, 2}\right)^{2}}{h_{i+\frac{1}{2}}} k_{2}$ & $\frac{\left(e_{i+1,3}-e_{i, 3}\right)^{2}}{h_{i+\frac{1}{2}}} k_{3}$ & $\frac{\left(e_{i+1,4}-e_{i, 4}\right)^{2}}{h_{i+\frac{1}{2}}} k_{4}$ \\
\hline 0 & 0 & 0.00056644 & 0.00106276 & 0.00106276 & 0.00056644 \\
\hline 1 & 0.2 & 0.00002116 & 0.00005625 & 0.00005625 & 0.00002116 \\
\hline$\cdots$ & .. & $\ldots$ & $\ldots$ & $\ldots$ & $\cdots$ \\
\hline 4 & 0.8 & 0.00017689 & 0.00036481 & 0.00036481 & 0.00017689 \\
\hline
\end{tabular}

Table 10. Error estimate of two-dimensional elliptic problem by using theorem 3 of Table 6.

\begin{tabular}{|c|c|c|c|c|c|}
\hline i & $x_{i}$ & $\frac{\left(e_{i, 2}-e_{i, 1}\right)^{2}}{k_{\frac{3}{2}}} h_{i}$ & $\frac{\left(e_{i, 3}-e_{i, 2}\right)^{2}}{k_{\frac{5}{2}}} h_{i}$ & $\frac{\left(e_{i, 4}-e_{i, 3}\right)^{2}}{k_{\frac{7}{2}}} h_{i}$ & $\frac{\left(e_{i, 5}-e_{i, 4}\right)^{2}}{k_{\frac{9}{2}}} h_{i}$ \\
\hline 0 & 0 & 0 & 0 & 0 & 0 \\
\hline 1 & 0.2 & 0.00007744 & 0 & 0.00007744 & 0.00080656 \\
\hline$\cdots$ & $\ldots$ & $\ldots$ & $\ldots$ & $\ldots$ & $\ldots$ \\
\hline 4 & 0.8 & 0.00003364 & 0 & 0.00003364 & 0.00017689 \\
\hline
\end{tabular}

Table 11. Error estimate of table (5) by using theorem (3) when $k^{0}=1$.

Now to estimate the error, equation (20) implies that $\mathrm{C}=0.2$ and $\mathrm{h}=0.2$. Then, we have

$\sum_{i, j} \frac{\left(e_{i+1, j}-e_{i, j}\right)^{2}}{h_{i+\frac{1}{2}}} k_{j}+\sum_{i, j} \frac{\left(e_{i, j+1}-e_{i, j}\right)^{2}}{k_{j+\frac{1}{2}}} h_{i} \leq 0.008$ and hence we have also, $\sum_{i, j}\left(e_{i, j}\right)^{2} h_{i} k_{j} \leq 0.008$

\begin{tabular}{|c|c|c|c|c|c|}
\hline i & $x_{i}$ & $\frac{\left(e_{i+1,1}-e_{i, 1}\right)^{2}}{h_{i+\frac{1}{2}}} k_{1}$ & $\frac{\left(e_{i+1,2}-e_{i, 2}\right)^{2}}{h_{i+\frac{1}{2}}} k_{2}$ & $\frac{\left(e_{i+1,3}-e_{i, 3}\right)^{2}}{h_{i+\frac{1}{2}}} k_{3}$ & $\frac{\left(e_{i+1,4}-e_{i, 4}\right)^{2}}{h_{i+\frac{1}{2}}} k_{4}$ \\
\hline 0 & 0 & 0.00001024 & 0.00001936 & 0.00001936 & 0.00001024 \\
\hline 1 & 0.2 & 0.00000144 & 0.00000324 & 0.00000324 & 0.00000144 \\
\hline$\ldots$ & $\cdots$ & $\cdots$ & $\cdots$ & $\ldots$ & $\cdots$ \\
\hline 4 & 0.8 & 0.0000009 & 0.00001764 & 0.00001764 & 0.0000009 \\
\hline
\end{tabular}

Table 12. Error estimate of two-dimensional elliptic problem by using theorem 3 of Table 7.

\begin{tabular}{|c|c|c|c|c|c|}
\hline i & $x_{i}$ & $\frac{\left(e_{i, 2}-e_{i, 1}\right)^{2}}{k_{\overline{2}}} h_{i}$ & $\frac{\left(e_{i, 3}-e_{i, 2}\right)^{2}}{k_{\frac{5}{2}}} h_{i}$ & $\frac{\left(e_{i, 4}-e_{i, 3}\right)^{2}}{k_{\frac{7}{2}}} h_{i}$ & $\frac{\left(e_{i, 5}-e_{i, 4}\right)^{2}}{k_{\frac{9}{2}}} h_{i}$ \\
\hline 0 & 0 & 0 & 0 & 0 & 0 \\
\hline 1 & 0.2 & 0.000001392 & 0 & 0.000001392 & 0.00001024 \\
\hline .. & $\ldots$ & $\ldots$ & $\ldots$ & $\ldots$ & $\ldots$ \\
\hline 4 & 0.8 & 0.00000144 & 0 & 0.00000144 & 0.000009 \\
\hline
\end{tabular}

Table 13. Error estimate of table (7) by using theorem () when $k^{0}=3$. 
Now to estimate the error, equation (14) implies that $\mathrm{C}=0.005$ and $\mathrm{h}=0.2$. then

$$
\sum_{i, j} \frac{\left(e_{i+1, j}-e_{i, j}\right)^{2}}{h_{i+\frac{1}{2}}} k_{j}+\sum_{i, j} \frac{\left(e_{i, j+1}-e_{i, j}\right)^{2}}{k_{j+\frac{1}{2}}} h_{i} \leq 0.0002
$$

hence, $\sum_{i, j}\left(e_{i, j}\right)^{2} h_{i} k_{j} \leq 0.0002$

\section{Conclusion}

In order to obtain a finite volume descritization, the domain $\Omega$ will be sub-divided in to many sub domains such that the collection of all those sub-domains forms a partition of $\Omega$. consider the second order one dimensional linear elliptic problems of the form as follow $u_{x x}(x)=-\sin x, x \in[0,1]$, with boundary conditions $u(0)=0, u(1)=0.017452406$. Here the exact solution of the problem is $u(x)=\sin x$, but the numerical solution over the control volume $\left(x_{i-\frac{1}{2}}, x_{i+\frac{1}{2}}\right), i=1,2, \ldots \ldots \ldots \ldots, N$, and by considering $h=0.05$ $20 u_{i+1}-40 u_{i}+20 u_{i-1}=\cos \left(x_{i+\frac{1}{2}}\right)-\cos \left(x_{i-\frac{1}{2}}\right), i=1,2,3, \ldots \ldots \ldots . . ., N$, where $N$ is natural number. Integration of the governing equation over a control volume to yield descritized equation at its nodal points.

\section{References}

[1] Herbin R. and O. Labergerie (2010), Finite volume schemes for elliptic and elliptic-hyperbolic Problems on triangular meshes, University of Chapman and Hall, Paris.
[2] Klaus's. 2010. Numerical Methods for Non-linear Elliptic Partial Differential Equation. Oxford University Press, New York.

[3] Morton, K. W. and E. Suli, 2003. Finite volume methods and their analysis, IMA J. Numer.

[4] Morton, K. W., Stynes M. and E. Suli (2005), Analysis of a cell-vertex finite volume method for a convection-diffusion problems, University of Albert, Canada.

[5] Evans, L. C. (1998), Partial Differential Equations, Providence: American Mathematical Society.

[6] Holubová, Pavel Drábek; Gabriela (2007). Elements of partial differential equations.

[7] Jost, J. (2002), Partial Differential Equations, New York: Springer-Verlag.

[8] Lewy, Hans (1957), "An example of a smooth linear partial differential equation without solution.

[9] Roubíček, T. (2013), Nonlinear Partial Differential Equations with Applications (2nd ed.).

[10] Solin, P. (2005), Partial Differential Equations and the Finite Element Method, Hoboken, NJ: J. Wiley \& Sons.

[11] Solin, P.; Segeth, K. \& Dolezel, I. (2003), Higher-Order Finite Element Methods, Boca Raton: Chapman \& Hall/CRC Press. 